\title{
Compreendendo o overtraining no desporto: da definição ao tratamento
}

\author{
Adelino S.R. Silva \\ Vanessa Santhiago \\ Cláudio A. Gobatto
}

https://doi.org/10.5628/rpcd.06.02.229

\author{
Universidade Estadual Paulista \\ Instituto de Biociências \\ Departamento de Educação Física \\ Rio Claro \\ São Paulo \\ Brasil
}

\section{RESUMO}

No desporto de alto nível o desenvolvimento de um programa de treinamento físico tem como principal objetivo a maximização da performance. Contudo, caso não ocorra uma periodização do treinamento adequada, os atletas podem desenvolver o fenômeno denominado overtraining ou sobretreino. O overtraining pode ser definido como um distúrbio neuroendócrino, que ocorre no eixo hipotálamo-hipófise, resultado do desequilíbrio entre a demanda do exercício e a capacidade de resposta do organismo. O objetivo do presente estudo de revisão é apresentar fatores inerentes ao overtraining, para que a comunidade científica aprofunde o conhecimento sobre este problema do treinamento e auxilie os profissionais do desporto e atletas a evitá-lo.

Palavras-chave: desporto, treinamento, overtraining.

\begin{abstract}
Understanding overtraining in sports: from definition to treatment

The main aim of a training program in the high level sport is to enhance performance. Athletes can present overtraining (OT) if an inadequate training periodisation occurs. Overtraining can be defined as a neuroendocrine disturb in hypothalamic-pituitary axis resulting from an unbalance between training and recovery. The aim of this review is to deepen the knowledge about overtraining factors, bringing actual scientific data to help coaches and athletes to deal with this problem, and overcome it.
\end{abstract}

Key Words: sport, training, overtraining. 


\section{INTRODUÇÃo}

No desporto de alto nível o desenvolvimento de um programa de treinamento físico tem como principal objetivo a maximização da performance. Contudo, para que ocorram adaptações fisiológicas e neurológicas positivas nos músculos e em outros tecidos é necessário que o treinamento apresente uma periodização que permita um equilíbrio entre a distribuição das cargas de treino e a recuperação do atleta. Durante e logo após uma sessão de treinamento ocorre uma fase catabólica, com diminuição da tolerância ao esforço, caracterizada por mudanças reversíveis de parâmetros bioquímicos, hormonais e imunológicos. Durante a recuperação, ocorre uma fase anabólica caracterizada por alta capacidade adaptativa e aumento das reservas energéticas, denominada supercompensação [3].

Em situações em que a periodização do treinamento não ocorre ou é mal planejada resultando em treinamento excessivo, os atletas podem desenvolver um fenômeno denominado overtraining (OT) ou sobretreino.

Embora muitos atletas e técnicos desportivos desconheçam este problema, alguns estudos demonstraram que o OT afeta aproximadamente $60 \%$ de corredores de fundo e meio fundo durante a carreira atlética [62], além de $50 \%$ de jogadores profissionais de futebol durante uma temporada competitiva de 5 meses [52] e 33\% de jogadores profissionais de basquete durante um período de treinamento de 6 semanas [87].

Dessa maneira este trabalho é de suma importância, pois através de uma revisão de literatura aprofundada visa apresentar fatores inerentes ao OT, para que a comunidade científica alargue o conhecimento sobre este problema do treinamento e auxilie os profissionais do desporto e atletas a evitá-la.

\section{OVERTRAINING}

O OT pode ser definido como um distúrbio neuroendócrino, que ocorre no eixo hipotálamo-hipófise, resultado do desequilíbrio entre a demanda do exercício e a capacidade de resposta do organismo [3].

\section{Diferença entre overtraining e overreaching}

No âmbito científico e prático é importante que esteja clara a distinção entre overtraining e overrea- ching (OR) para que tanto os pesquisadores, quanto os treinadores e fisiologistas, possam identificar os sintomas em seus atletas e tomar as providências necessárias. De acordo com Lehmann, Foster e Keul [48], o OR ocorre após vários dias de treinamento intenso e está associado à fadiga muscular ou periférica, podendo ser definido como uma fase pré-overtraining [44]. A recuperação do atleta ocorre após alguns dias de afastamento dos treinamentos ou diminuição das cargas de treino.

Já o OT tem maior relação com a fadiga central e a recuperação pode demorar semanas ou meses. $\mathrm{O}$ atleta que se encontra em OT, normalmente, apresenta redução de performance acompanhada por alterações fisiológicas, psicológicas e bioquímicas.

\section{Formas de overtraining}

Segundo Israel [36], o OT pode ser classificado em duas categorias: a parassimpática e a simpática. A forma simpática ou clássica do OT é caracterizada pelo aumento da atividade do sistema nervoso simpático em repouso. O sistema nervoso simpático provoca alterações básicas das funções do organismo, facilitando a resposta motora ao estresse agudo ou à atividade física. Ocorre com maior freqüência em equipes que utilizam predominantemente o metabolismo anaeróbio alático e lático para suprir as demandas musculares, além de ser influenciada pelo estado emocional do atleta que não depende apenas de fatores estressantes relacionados à modalidade, mas também aos fatores extra-treinamento.

Já a forma parassimpática do OT é caracterizada pela predominância do tônus parassimpático no repouso, assim como durante o exercício, e é observada com maior freqüência em atletas de endurance [3].

\section{Modelos para estudar o overtraining e overreaching}

De acordo com Mackinnon [56], dois modelos gerais são utilizados para o estudo do OT. No primeiro, os atletas são avaliados durante a temporada competitiva e as respostas fisiológicas e psicológicas são comparadas para cada atleta entre períodos de alta e baixa intensidade de treinamento, ou entre atletas que demonstraram sintomas de OT e os que não apresentaram tais sintomas. A vantagem deste método é que os atletas são avaliados no seu ambiente natural, sem a manipulação do regime normal de 
treinamento. A desvantagem é a dificuldade de se controlar as variáveis que podem ser alteradas devido à alimentação, variabilidade climática e estresse competitivo.

No segundo modelo, o treinamento é intensificado propositadamente durante um período de 4 semanas. Por razões éticas, quatro semanas é o período máximo que os atletas podem suportar o aumento de cargas que já são intensas [52, 57]. A performance aeróbia, anaeróbia lática ou alática e as variáveis psicológicas e fisiológicas são comparadas antes e após o período de treinamento intensificado ou entre atletas que demonstraram sintomas de overreaching, já que 4 semanas são consideradas um período curto para levar o atleta ao overtraining, e os que não apresentaram tais sintomas. Este método possibilita um melhor controle das variáveis que possam confundir o diagnóstico.

Contudo, o aumento das cargas de treinamento é superior ao que normalmente ocorre, não refletindo, desta maneira, o programa de treinamento do atleta. Exceto estas limitações, ambos os modelos fornecem informações úteis para identificar os marcadores e compreender os mecanismos responsáveis pelo overtraining ou overreaching.

\section{Etiologia do overtraining}

A tolerância do atleta ao estresse é determinada pela sua capacidade de adaptação, estratégias de competição e características fisiológicas. A quantidade total de fatores estressantes internos e externos determina a maior vulnerabilidade de alguns atletas. Em relação aos fatores internos, Koutedakis e Sharp [43] verificaram que homens e mulheres respondem de maneira diferente aos fatores de estresse, e que a incidência do OT parece ser maior nos homens.

Sobre os fatores externos, um aumento progressivo no volume do treinamento intenso, com considerável aumento no volume total de treinamento parece ser a causa predominante do aumento da suscetibilidade ao OT.

Existem muitas teorias, embora nenhuma delas conclusivas, sobre a origem e mudanças fisiopatológicas do estado de OT. Possivelmente os fatores responsáveis pela síndrome do sobretreino relacionados à adaptação central são:

1) Desequilíbrio circulante de aminoácidos. Durante o exercício aeróbio de longa duração pode ocorrer uma diminuição da concentração dos aminoácidos de cadeia ramificada (AACR; leucina, isoleucina e valina) devido à oxidação pelo músculo esquelético para a ressíntese de adenosina trifosfato (ATP).

Simultaneamente ocorre um aumento da concentração de aminoácidos aromáticos (AAA; tirosina, fenilanina e triptofano). O triptofano (Trp) é o precursor da serotonina no cérebro e $90 \%$ deste aminoácido circula ligado à albumina, o restante circula livremente $\left(\operatorname{Trp}_{\mathrm{L}}\right)$ [71].

Assim como os aminoácidos de cadeia ramificada (AACR), os ácidos graxos livres (AGLs) também são oxidados pelo músculo esquelético para produzir ATP quando ocorre depleção do glicogênio muscular e hepático, respectivamente. Os AGLs, como não são solúveis em água, também utilizam a albumina para circularem no sangue. Desta maneira, ocorre uma competição pela albumina entre os AGLs e o $\operatorname{Trp}_{\mathrm{L}}$, e quanto maior for a utilização de AGLs para a ressíntese de ATP maior será a quantidade de $\operatorname{Trp}_{\mathrm{L}}$ [50]. $\mathrm{O}$ transporte dos AACR e dos AAA pela barreira hematoencefálica ocorre pelo mesmo mecanismo específico, que é controlado por competição, e a afinidade do transportador pelo aminoácido é determinada pelas concentrações dos demais aminoácidos. Como há baixa concentração de AACR e alta concentração de AAA, principalmente o $\operatorname{Trp}_{\mathrm{L}}$, este aminoácido chega ao cérebro para formar a serotonina [75]. Este fenômeno já foi observado em atletas em OT [26] e uma diminuição na concentração sanguínea da razão $\operatorname{Tr}_{\mathrm{L}} / \mathrm{AACR}$ tem sido proposta como ferramenta para diagnosticar OT em atletas de endurance [64].

2) Variações no eixo hipotálamo-hipófise-adrenal (HHA). Duclos et al [16], observaram que a repetição do estresse fisiológico imposto pelo treinamento físico em corredores de longa distância influenciava na produção do hormônio adrenocorticotrópico (ACTH). Na realidade, ocorria um aumento da concentração plasmática deste hormônio, contudo não foi observada alteração na concentração plasmática do principal hormônio da sua glândula alvo, ou seja, o cortisol. Isto poderia ser explicado através da diminuição da sensibilidade da glândula adrenal a estimulação do ACTH e/ou a uma diminuição da sensibilidade do eixo hipotálamo-hipófise ao feedback negativo imposto pelo cortisol. 
Posteriormente Duclos et al [15], estimularam o eixo hipotálamo-hipófise-adrenal através de administração farmacológica e a glândula adrenal com ACTH, concluindo que a concentração normal de cortisol em resposta ao aumento do ACTH induzido pelo estresse físico é, supostamente, resultado da diminuição da sensibilidade da hipófise para o feedback negativo do cortisol e não da glândula adrenal ao ACTH.

Luger et al [55] verificaram em atletas em OT com hipercortisolismo médio uma diminuição na resposta do cortisol e do ACTH ao hormônio liberador da corticotropina $(\mathrm{CRH})$, mas um aumento normal em resposta ao exercício físico, sugerindo que o estresse imposto pela atividade física sobrepõe o controle do feedback negativo exercido pelo aumento da concentração de cortisol.

3) Disfunção do sistema nervoso autônomo. A excreção urinária noturna de catecolaminas parece refletir a atividade intrínseca do sistema nervoso simpático [41]. Alguns autores têm verificado uma diminuição da excreção urinária noturna de catecolaminas em atletas em overtraining [52, 53]. Além disso, Steinle [81] encontrou uma correlação negativa entre a excreção de catecolaminas e a latência da fase REM do sono $(r=-0,46 ; p<0,01)$, confirmando a hipótese de que uma diminuição na excreção de catecolaminas pode indicar a presença de fadiga central.

Com relação à concentração plasmática de catecolaminas, em atletas em overtraining, tem se observado um aumento nos níveis de noradrenalina plasmática em repouso e em resposta ao exercício de intensidade moderada [46, 49]. Hooper et al [34] também verificaram aumento nas concentrações plasmáticas de noradrenalina no repouso, em nadadores em OT. Quanto à adaptação periférica, a capacidade dos órgãos de receberem informação do sistema nervoso central parece ser influenciada pelo overtraining [53]. Por exemplo, Lehmann, Foster e Keul [48] concluíram que a implantação de um programa de treinamento diminuiu a sensibilidade da glândula adrenal ao ACTH em atletas recreacionais. Isto poderia explicar alguns estudos que indicaram uma diminuição na liberação do cortisol em atletas em OT $[4,49,84]$.

\section{Estresse oxidativo e overtraining}

Os radicais livres de oxigênio (RLO) são produzidos naturalmente pelo nosso organismo e muitas vezes são de extrema utilidade, como na ativação do sistema imunológico, no processo de desintoxicação de drogas e na produção do fator relaxante derivado do endotélio (óxido nítico), que é fundamental para o relaxamento dos vasos sangüíneos [38].

Cerca de 2 a $5 \%$ do oxigênio total consumido dá origem a produtos reduzidos como o radical ânion superóxido, peróxido de hidrogênio e radicais hidroxila. Essas espécies de subprodutos são coletivamente chamadas de espécies reativas de oxigênio (EROs) [38]. Altos níveis de EROs são responsáveis por várias ações deletérias, como a oxidação de estruturas celulares e o prejuízo na homeostase intracelular. Com o objetivo de diminuir a ação tóxica da EROs, o organismo dispõe do sistema enzimático antioxidante, no qual as enzimas parecem possuir a capacidade de se adequar ao aumento da produção da EROs através do aumento na sua atividade [72]. Além disso, várias moléculas com ação antioxidante também são consumidas na dieta, como $\alpha$-tocoferol (vitamina E), $\beta$-caroteno (precursor de vitamina $A$ ), selênio, zinco, cobre, glutationa reduzida (GSR) e ácido ascórbico [89].

Embora essas defesas antioxidantes reduzam o risco de lesões oxidativas da EROs, hábitos de vida inapropriados, tais como, o consumo excessivo de álcool, hábito de fumar, dieta inadequada, exposição freqüente à radiação não ionizante UV, à poluição, além do estresse emocional [18], do envelhecimento [14] e da prática constante de exercício extenuante [18] podem desencadear um desequilíbrio entre a defesa antioxidante e a produção da EROs gerando uma situação de estresse oxidativo.

O exercício físico está associado ao aumento de radicais livres devido ao aumento do consumo de oxigênio pelos tecidos ativos [12, 91]. Bloomer e Goldfard [6] verificaram que após o exercício físico agudo ou crônico existe um aumento da concentração de radicais livres nos tecidos biológicos que coincide com a presença de danos teciduais.

$\mathrm{Na}$ realidade, a relação entre estresse oxidativo e exercício físico está diretamente relacionada à intensidade e duração do exercício. O exercício físico intenso normalmente desencadeia o estresse oxidativo que gera diminuição do desempenho físico, fadiga 
muscular, danos musculares e, até, overtraining [42], promovendo alteração do sistema imune e do estado de treinamento dos indivíduos [2].

De acordo com Lamprecht et al [45], os danos musculares causados pelo estresse oxidativo são mais acentuados em indivíduos pouco treinados que realizam exercícios com intensidade e duração acima do seu condicionamento físico. Miyazaki et al [61] verificaram que a realização de um treinamento moderado, antes dos indivíduos serem submetidos ao exercício agudo de alta intensidade, minimiza os efeitos do estresse oxidativo.

A prática regular de atividade física moderada altera positivamente a homeostase oxidativa de células e tecidos, através da diminuição dos danos oxidativos e do aumento da resistência ao estresse oxidativo $[12,13]$. Dessa forma são desejados como resposta adaptativa a um treinamento eficiente, altos níveis de defesa antioxidante e baixos níveis de produção do estresse oxidativo [91].

\section{Hipótese das citocinas e overtraining}

As citocinas são pequenas proteínas ou peptídeos que compreendem um grupo muito extenso de moléculas envolvidas na emissão de sinais entre as células, através de um processo análogo ao dos hormônios, durante o desencadeamento das respostas imunes. As citocinas podem ser divididas, de acordo com sua estrutura ou função, em diversas categorias: interferons (IFN), interleucinas (IL-1 a IL-15), fator estimulador de colônias (CSF), fator de necrose tumoral (TNF- $\alpha$ e TNF- $\beta$ ), e fator de transformação de crescimento (TGF- $\beta$ ) [79].

Geralmente, as citocinas são estudadas de acordo com sua função pró ou antiinflamatória. As citocinas pró-inflamatórias incluem as IL-1 $\beta$, IL-6 e IL8 e o TNF- $\alpha$. Também existe uma quantidade de citocinas antiinflamatórias cujo único propósito é regular o processo inflamatório. Algumas dessas citocinas incluem IL-4, IL-10, IL-13, assim como, o receptor antagonista da IL-1 (IL-1ra) [79].

Com relação ao exercício físico, as IL-1 $1 \beta$ e IL-6 e o TNF- $\alpha$ são as citocinas mais estudadas. Segundo Pedersen, Rohde e Ostrowski [69], a maioria dos estudos avaliam o comportamento dessas citocinas após a realização do esforço físico. O aumento da IL6 em resposta ao exercício físico foi constatado por diversos autores $[67,82,85]$. No entanto, os resultados em relação à resposta TNF- $\alpha$ após o exercício são contraditórios. Alguns autores não observaram mudanças [74, 82], enquanto outros constataram aumento desse tipo de citocina $[67,68]$.

$\mathrm{O}$ aumento das citocinas em resposta ao exercício físico ocorre principalmente nas atividades em que a contração é excêntrica. Bruunsgaard et al [9] compararam o efeito da contração excêntrica e concêntrica, com o mesmo consumo de oxigênio, na concentração de catecolaminas, citocinas e de creatina quinase (CK). Embora os níveis de catecolaminas não tenham apresentado diferença, o nível de creatina quinase aumentou cerca de 40 vezes, 4 dias após a atividade excêntrica.

No exercício concêntrico não houve alteração na concentração de CK. A concentração de IL-6 aumentou 5 vezes após o exercício excêntrico e apresentou correlação significativa com a concentração de CK. Este estudo indica que existe uma relação entre a concentração de IL-6 e microlesão muscular. Muitos autores sugerem que as lesões musculoesqueléticas podem ser consideradas como uma manifestação muscular da síndrome de sobretreino [21, 65]. Seene et al [76], através de biópsias musculares, verificaram um grande número de lesões em atletas em overtraining.

Smith [79] sugere que as lesões musculares não só ocorrem nos atletas em sobretreino, como são a causa do OT. A formação de microlesões musculares em resposta ao volume e/ou intensidade de treinamento associadas com recuperação insuficiente seriam a principal causa do overtraining. Através dessa linha de raciocínio o autor sugere que a maioria dos sintomas fisiológicos e psicológicos do OT podem surgir de uma lesão muscular.

Contudo, recentemente, Halson et al [30] não verificaram nenhuma alteração na concentração plasmática de IL- 6 e TNF- $\alpha$ após um período de treinamento intenso de 2 semanas em que houve diminuição da performance e aumento dos distúrbios de humor.

\section{Diagnóstico do overtraining}

Embora muitos estudos tenham sido realizados com o objetivo de determinar parâmetros capazes de detectar o OT, ainda não foi possível estabelecer um marcador universal capaz de identificar, em um 
grupo de atletas, aqueles que apresentam possibilidade de desenvolver o OT ao longo de um programa de treinamento [22, 33, 47, 84]. Desta maneira, o monitoramento regular da combinação de variáveis de performance, fisiológicas, psicológicas, bioquímicas e imunológicas parece ser a melhor estratégia para avaliar a adaptação do atleta ao treinamento e evitar o OT.

Dentre a grande quantidade de variáveis citadas em estudos de overtraining podemos classificá-las em alguns parâmetros:

\section{1) Parâmetros de performance}

a) Consumo máximo de oxigênio $\left(\mathrm{VO}_{2} \max \right)$ : Alguns autores verificaram que o $\mathrm{VO}_{2}$ max em teste incremental até à exaustão diminuía nos atletas em OT $[49,80,84]$ enquanto outros não verificaram nenhuma alteração $[22,83]$.

b) Freqüência Cardíaca (FC): A FC em repouso parece não apresentar alteração em atletas em OT [33, 51]. Por outro lado, uma série de estudos indica que a FC máxima diminui 5-10 bpm [49, 51, 90].

c) Lactato Sanguíneo: Muitos autores, em diferentes modalidades esportivas, verificaram que a concentração pico de lactato sanguíneo ([Lac $]_{\text {pico }}$ ) diminui em atletas em overtraining [11, 31, 83]. Neste tipo de atleta, as concentrações de lactato sanguíneo durante exercício incremental de intensidade moderada ([Lac]) também diminuem, refletindo um aumento no cálculo do Limiar Anaeróbio (Lan) que mascara a real condição aeróbia do atleta $[5,35,37,39]$. Na tentativa de eliminar esta contradição, Snyder et al [80] propuseram complementar a mensuração do lactato sanguíneo com as taxas de percepção de esforço (RPE). Assim, a razão [Lac]/RPE diminuiria nos atletas em OT e permaneceria inalterada quando ocorresse adaptação positiva ao treinamento. Este mecanismo foi considerado eficiente apenas para atletas em OR. Bosquet, Léger e Legros [7], conseguiram corrigir a superestimação do Lan em atletas em OR e OT ao utilizar a $[\mathrm{Lac}]$ como $\%[\mathrm{Lac}]_{\text {pico }}$.

\section{2) Parâmetros imunológicos}

a) Leucócitos: Normalmente, a quantidade de leucócitos permanece dentro dos valores de referência, mesmo durante períodos de treinamento físico intenso e em atletas em OT [27, 34, 58]. Contudo,
Lehmann et al [51] observaram em corredores uma diminuição da quantidade de leucócitos abaixo dos valores de referência, em resposta a um período de quatro semanas em que o volume, e não a intensidade de treinamento, foi dobrado. Matvienko [59] verificou uma diminuição da contagem de leucócitos em atletas com estagnação da performance, mas sem sintomas de OT.

b) Linfócitos: Assim como os leucócitos, o número de linfócitos permanece dentro dos valores de referência em resposta ao treinamento físico intenso ou em atletas em OT [25, 27]. Hooper et al [33] não verificaram diferença significativa para contagem de linfócitos entre o grupo de nadadores normal e os classificados em OT durante uma temporada de seis meses. c) Neutrófilos: Alguns estudos têm registrado, em modalidades esportivas distintas, uma diminuição da atividade basal e pós-exercício dos neutrófilos de atletas comparados com sedentários ou dos mesmos atletas em diferentes fases do treinamento [29, 73, 78]. Com relação à contagem de neutrófilos, Hooper et al [33] não verificaram diferença significativa entre o grupo de nadadores normal e os classificados em OT durante uma temporada de seis meses, contudo houve diferença significativa entre os atletas em OT durante o polimento.

d) Eosinófilos: Gabriel et al [25] verificaram uma menor quantidade de eosinófilos nos atletas em OT. Fry et al [22] também observaram, em atletas em OT, uma diminuição na contagem de eosinófilos antes do início do programa de treinamento intenso.

\section{3) Parâmetros hormonais}

a) Testosterona: Flynn et al [20] verificaram uma diminuição da concentração de testosterona plasmática, assim como da performance, em nadadores após o aumento de $88 \%$ do volume de treinamento, durante duas semanas. Sobre o exercício resistido, recentemente Durand et al [17] concluíram que, tanto a contração dinâmica excêntrica. quanto a concêntrica aumentaram significativamente as concentrações plasmáticas de T e TF.

b) Cortisol: Muitos autores não verificaram nenhuma alteração na concentração plasmática basal de cortisol em atletas em OT [33, 83, 84], enquanto outros observaram um aumento $[1,4]$, diminuição [49], ou respostas variáveis [31]. 
c) Razão Testosterona/Cortisol (T/C): Adlercreutz et al [1] propuseram que a diminuição superior a $30 \%$ na razão $\mathrm{T} / \mathrm{C}$ indicaria o estado de OT. Embora alguns estudos tenham constatado alterações na razão $\mathrm{T} / \mathrm{C}$ durante períodos de treinamento intenso $[40,88]$, a maioria dos pesquisadores não verificou tais alterações em uma série de atletas incluindo corredores $[20,23,86]$ e nadadores [20, 33, 40]. Além disso, alguns estudos verificaram queda igual ou superior a $30 \%$ na razão $\mathrm{T} / \mathrm{C}$, que correspondeu com ganho de performance [19, 32]. Gorostiaga et al [28] sugerem que uma queda inferior a $45 \%$ da razão $\mathrm{T} / \mathrm{C}$ nem sempre pode indicar OT.

d) Catecolaminas: Mudanças na concentração plasmática e excreção urinária da noradrenalina têm sido associadas com o OT [58]. Aumento e diminuição da concentração plasmática em repouso e após exercício de alta intensidade, respectivamente, foram registrados em corredores de meio fundo após períodos de treinamento intenso [46, 49, 51, 58]. Hooper et al [33] também constataram menor concentração plasmática de noradrenalina em nadadores em OT.

\section{4) Parâmetros hematológicos}

a) Hematócrito: Mackinnon et al [58] observaram uma tendência do hematócrito de diminuir em nadadoras em OT, após duas e quatro semanas de aumento progressivo no volume de treinamento. b) Eritrócitos: Mackinnon et al [58] verificaram uma diminuição da quantidade de eritrócitos durante quatro semanas de aumento progressivo no volume de treinamento em nadadores. Este declínio, aproximadamente $8-12 \%$, foi similar, em magnitude, ao declínio da concentração de hemoglobina, em valores de 5-9\%. Outros autores também constataram diminuição nas concentrações de eritrócitos e hemoglobinas após treinamento de alta intensidade [63, 77].

c) Creatina quinase (CK). Halson et al [30], após um período de seis semanas de treinamento com ciclistas, com a terceira e quarta e a quinta e sexta semanas apresentando o dobro e a metade do volume das duas primeiras semanas, respectivamente, observaram um aumento significativo na atividade da $\mathrm{CK}$ ao final da terceira e quarta semanas de treinamento. d) Uréia. A maioria dos estudos não constatou alteração na concentração plasmática de uréia em atletas em OT [44, 47, 49]. Halson et al [30] verificaram uma tendência $(p=0,057)$ da concentração de uréia aumentar após duas semanas em que o volume de treinamento normal em ciclistas foi dobrado.

e) Amônia: Leitzmann, Jung e Seiler [54] verificaram uma diminuição na concentração de amônia nos atletas em OT. Contudo, Halson et al [30] verificaram uma tendência $(\mathrm{p}=0,067)$ da concentração de amônia aumentar após duas semanas em que o volume de treinamento normal em ciclistas foi dobrado. f) Creatinina: Lehmann et al [47] não observaram alteração na concentração plasmática basal de creatinina em corredores de fundo e meio fundo em resposta ao aumento semanal de aproximadamente $33 \%$ no volume de treinamento durante 4 semanas.

\section{5) Parâmetros psicológicos}

Alguns estudos têm verificado uma relação diretamente proporcional entre os distúrbios de humor e as cargas de treinamento $[20,62,66]$.

O questionário POMS (Profile of Mood States), desenvolvido em 1971 por MacNair, Lorr e Droppleman [60] é um dos mais conhecidos instrumentos para estimar estados emocionais e os dados subjetivos acerca dos sentimentos, afetos e humor [8]. Recentemente, Peluso [70] traduziu e validou o questionário POMS para o português. Os atletas em OT, normalmente, apresentam aumento das variáveis negativas e diminuição da variável positiva do POMS $[19,20,66]$.

\section{Prevenção e tratamento}

A prevenção do OT inclui uma periodização do treinamento que permita intercalar sessões de treinamento com repouso adequado. Além disso, é fundamental a execução de testes ao longo da periodização que possibilitem a detecção precoce do OT. Contudo, a interpretação dos resultados obtidos na bateria de testes deve ser conduzida de forma consistente, para que se evite confundir a fadiga normal do treinamento com o OT [24].

Segundo Budget [10] o tratamento mais adequado para o atleta que está em overtraining é o repouso, que em geral não deve ser inferior a cinco semanas, com sessões crescentes de atividade aeróbia. Contudo, a completa recuperação pode levar doze semanas e a individualidade biológica de cada atleta deve ser respeitada. 


\section{AGRADECIMENTOS}

Apoio financeiro: FAPESP (processo 03/04214-3); CNPq (processo 130441/2004-0); Fundunesp (processo 00844/03-DFP).

\section{CORRESPONDÊNCIA}

\section{Adelino Sanchez Ramos da Silva}

Rua Rui Barbosa ${ }^{\circ} 221$

Vila Monteiro

13560-330, São Carlos

São Paulo

Brasil

adelinosanchez@hotmail.com

\section{REFERÊNCIAS BIBLIOGRÁFICAS}

1. Adlercreutz H, Harkonen M, Kuopassalmi K, Karvonen J (1986). Effect of training on plasma anabolic and catabolic steroid hormones and their response during physical exercise. Int J Sports Med 7: 27-28

2. Alessio HM, Hagerman AE, Fulkerson BK, Ambrose J, Rice RE, Wiley RL (2000). Generation of reactive oxygen species after exhaustive aerobic and isometric exercise. Med Sci Sports Exerc 32:1576-1581

3. Baptista CAS, Ghorayeb N, Dioguardi GS (1999). Sobretreinamento. In Ghorayeb N, Barros T (ed). O Exercício. São Paulo: Atheneu, 313-320

4. Barron GL, NoakesTD, Levy W, Smith C, Millar RP (1985). Hypothalamic dysfunction in overtrained athletes. J Clin Endocrinol Metab 60: 803-806

5. Billat V (1996). Use of blood lactate measurements for prediction of exercise performance and for control of training. Sports Med 22: 803-806.

6. Bloomer RJ, Goldfarb AH (2004). Anaerobic exercise and oxidative stress: a review. Can J Appl Physiol 29: 245-263.

7. Bosquet L, Leger L., Legros P (2001). Blood Lactate Response to overtraining in male athletes. Eur J Appl Physiol 84: 107-114.

8. Brandão MRF (1999). Psicologia do Esporte. In Ghorayeb N, Barros T (ed). O Exercício. São Paulo: Atheneu, 239-245.

9. Bruunsgaard H, Galbo H, Halkjaer-Kristensen J, Johansen TL, MacLean DA, Pedersen BK (1997). Exercise-induced increase in interleukin- 6 is related to muscle damage. $J$ Physiol (London) 499:833-841.

10. Budjet R (1994). The overtraining syndrome. Br Med J 309: 465-468

11. Callister R, Callister RG, Fleek SJ, Dudley GA (1990). Physiological and performance responses to overtraining in elite judo athletes. Med Sci Sports Exerc 22: 816-824

12. Cooper CE, Vollaard NB, Choeiri T, Wilson MT (2002). Exercise, free radicals and oxidative stress. Biochem Soc Trans 30: 280-285

13. Di Meo S, Venditti P (2001). Mitochondria in exerciseinduced oxidative stress. Biol Signal Recept 10:125-140

14. Droge W (2002). Free radicals in the physiological control of cell function. Physiol Rev 82:47-95

15. Duclos M, Corcuff JB, Arsac L, Moreau-Gaundry F, Rashedi M, Roger P, Tabarin A, Manier G (1998). Corticotroph axis sensitivity after exercise in endurancetrained athletes. Clin Endocrinol 48: 493-501

16. Duclos M, Corcuff JB, Rashedi M, Fougere V, Manier G (1997). Trained versus untrained men: different hypothalamo-pituitary adrenal axis response to exercise recovery. Eur J Appl Physiol 75: 343-350

17. Durand RJ, Castracane D, Hollander DB, Tryniecki JL, Bamman MM, O`neal S, Herbert EP, Kraemer RR (2003). Hormonal responses from concentric and eccentric muscle contractions. Med Sci Sports Exerc 35: 937-943

18. Elsayed NM (2001). Antioxidant mobilization in response to oxidative stress: a dynamic environmental-nutritional interaction. Nutrition 17 828-834

19. Filaire E, Bernain X, Sagnol M, Lac G (2001). Preliminary results on mood state, salivary testosterone: cortisol ratio and team performance in professional soccer team. Eur J Appl Physiol 86: 179-184

20. Flynn MG, Pizza FX, Boone JBJr, Andres FF, Michaud TA, Rodrigez-Zayas JR (1994). Indices of training stress during 
competitive running and swimming seasons. Int $J$ Sports Med 15: 21-26

21. Fry AC (1998). The role training intensity in resistance exercise-overtraining and overreaching. In Kreider RB, Fry AC, O’Toole ML (ed). Overtraining in Sport. Champaign, IL: Human Kinetics, 107-130

22. Fry RW, Morton AR, Garcia-Webb P, Crawford GPM, Keast D (1992). Biological responses to overload training in endurance sports. Eur J Appl Physiol 64: 335-344

23. Fry RW, Morton AR, Keast D (1991). Overtraining in athletes, an update. Sports Med 12: 32-65

24. Fry RW, Morton AR, Keast D (1992). Periodisation of training stress, a review. Can J Sport Sci 17: 234-240

25. Gabriel HH, Urhausen A, Valet G, Heidelbach U, Kindermann W (1998). Overtraining and immune system: A prospective longitudinal study in endurance athletes. Med Sci Sports Exerc 30: 1151-1157

26. Gastmann UA, Lehmann MJ (1998). Overtraining and the BCAA hypothesis. Med Sci Sports Exerc 30:128-139

27. Gleeson M, McDonald WA, Cripps AW, Pyne DB, Claney RL, Fricker PA (1995). The effect on immunity of long term intensive training on elite swimmers. Clin Exp Immunol 102: 210-216

28. Gorostiaga EM, Izquierdo M, Rusta M, Iribarren J, González-Badillo JJ, Ibánez J (2004). Strength training effects on physical performance and serum hormones in young soccer players. Eur J Appl Physiol 91: 698-707

29. Hack V, Strobel G, Weiss MN, Weicker H (1994). PMN cell counts and phagocytic activity of highly trained athletes depend on training period. J Appl Physiol 77: 1731-1735

30. Halson LS, Lancaster GI, Jeukendrup AE, Gleeson M (2003). Immunological responses to overreaching in cyclists. Med Sci Sports Exerc 35: 854-861

31. Hedelin R, Kenttá G, Wiklund U, Bjerle P, Henrisson-Larsè K (2000). Short-term overtraining: effects on performance, circulatory responses, and heart rate variability. Med Sci Sports Exerc 32: 1480-1484

32. Hoogeveen AR, Zonderland ML (1996). Relationships between testosterone, cortisol, and performance in professional cyclists. Int $J$ Sports Med 17: 423-428

33. Hooper SL, Mackinnon LT, Gordon RD, Bachmann AW (1993). Hormonal responses of elite swimmers to overtraining. Med Sci Sports Exerc 25: 741-747

34. Hooper SL, Mackinnon LT, Howard A, Gordon RD, Bachmann AW (1995). Markers for monitoring overtraining and recovery in elite swimmers. Med Sci Sports Exerc 27: 106-112

35. Hurley BF, Hagberg JM, Allen WK, Seals DR, Young JC, Cuddihee RW, Holloszy JO (1984). Effect of training on blood lactate levels during submaximal exercise. J Appl Physiol 56: 1260-1264

36. Israel SZ (1976). Problematik des übertraings aus internisistischer und leistungs physiologischer sicht. Med Sport 16: $1-12$

37. Jacobs I (1986). Blood lactate: implications for training and sports performance. Sports Med 3: 10-25

38. Jenkins RR, Goldfarba A (1993). Introduction: oxidant stress, aging and exercise. Med Sci Sports Exerc 25: 210-212

39. Jeukendrup AE, Hessellink MKC, Snyder AC, Kuipers $H$, Keizer HA (1992). Physiological changes in male competitive cyclists after two weeks of intensified training. Int $J$ Sports Med 13: 534-541
40. Kirwan JP, Costill DL, Fynn MG, Mitchell JB, Fink WJ, Neufer PD, Houmard JA (1988). Physiological responses to successive days of intense training in competitive swimmers. Med Sci Sports Exerc 20: 255-259

41. Kjaer N, Secher H, Bach FW, Sheikh S, Galbo H (1989). Hormonal and metabolic responses to exercise in humans: effect of sensory nervous blockade. Am J Physiol 257: 95-100

42. Konig D, Wagner KH, Elmadfa I, Berg A (2001). Exercise and oxidative stress: significance of antioxidants with reference to inflammatory, muscular, and systemic stress. Exerc Immunol Rev 7:108-133

43. Koutedakis Y, Sharp CC (1998). Seasonal variations of injury and overtraining in elite athletes. Clin J Sports Med 8: 18-21

44. Kuipers H, Keizer HA (1988). Overtraining in elite athletes: review and directions for the future. Sports Med 6: 79-92

45. Lamprecht M, Greilberger J, Oetti K (2004). Analytical aspects of oxidatively modified substances in sports and exercises. Nutrition 20: 728-730

46. Lehmann M, Baumgartl P, Weisenack C, Seidel A, Baumann H, Fischer S, Spori U, Gendrisch G, Kaminski R, Keul J (1992). Training-overtraining: influence of a defined increase in training volume vs training intensity on performance, catecholamine and some metabolic parameters in experienced middle- and long-distance runners. Eur J Appl Physiol 64: 169-177

47. Lehmann M, Dickhuth HH, Gendrisch G, Lazar W, Thum M, Kaminsky R, Aramend JF, Peterke E, Wieland W, Keul J (1991). Training-overtraining: a prospective, experimental study with experienced middle and long distance runners. Int J Sports Med 12: 444-452

48. Lehmann M, Foster C, Keul J (1993). Overtraining in endurance athletes: a brief review. Med Sci Sports Exerc 25: 854-862

49. Lehmann M, Gastmann U, Petersen KG, Bachl N, Seidel A Khalaf AN, Fischer S, Keul J (1992). Training-overtraining: performance and hormonal levels after a defined increase in training volume vs. intensity in experienced middle and long-distance runners. Br J Sports Med 26: 233-242

50. Lehmann M, Jakob E, Gastmann U, Steinacker JM, Keul J (1995). Unaccustomed high mileage compared to intensity training-related neuromuscular excitability in distance runners. Eur J Appl Physiol 70: 457-461

51. Lehmann M, Mann H, Gastmann U, Keul J, Vetter D, Steinacker JM, Haussinger D (1996). Unaccustomed high-mileage vs intensity training-related changes in performance and serum amino acid levels. Int J Sports Med 17: 187-192

52. Lehmann M, Schnee W, Scheu R, Stockhausen W, Bachl N (1992). Decreased nocturnal catecholamine excretion: Parameter for an overtraining syndrome in athletes? Int $J$ Sports Med 13: 236-242

53. Lehmann M, Wieland H, Gastmann U (1997). Influence of an unaccustomed increase in training volume vs intensity on performance, hematological and blood-chemical parameters in distance runners. J Sports Med Phys Fitness 37: $110-116$

54. Leitzmann L, Jung K, Seiler D (1991). Effect of an extreme physical endurance performance on selected plasma proteins. Int J Sports Med 12: 100 
55. Luger A, Deuster PA., Kyle SB, Gallucci WT, Montgomery LC, Gold PW, Lynn-Loriaux D, Chrousos GP (1987). Acute hypothalamic-pituitary-adrenal responses to the stress of treadmill exercise. $N$ Engl J Med 316: 1309-1315

56. Mackinnon LT (2000). Overtraining effects on immunity and performance in athletes. Immunol Cell Biol 78: 502-509

57. Mackinnon LT, Hooper SL (1996). Plasma glutamine and upper respiratory tract infection during intensified training is swimmers. Med Sci Sports Exerc 28: 285-90

58. Mackinnon LT, Hooper SL, Jones S, Gordon RD, Bachmann A (1997). Hormonal, immunological, and hematological responses to intensified training in swimmers. Med Sci Sports Exerc 29: 1637-1645

59. Matvienko LA (1981). A study of peripheral blood in track and field athletes. Sov Sports Rev 16: 50-51

60. McNair DM, Lorr M, Droppleman LF (1971). Profile of mood states manual. San Diego: Educational and Industrial Testing Services

61. Miazaki H, Oh-ishi S, Ookawara T, Kizaki T, Toshinai K, Ha S, Haga S, Ji LL, Ohno H (2001). Strenuous endurance training in humans reduce oxidative stress following exhausting exercise. Eur J Appl Physiol 84:1-6

62. Morgan WP, Brown DR, Raglin JS, O'Connor PJ, Ellickson KA (1987). Physiological monitoring of overtraining and staleness. Br J Sports Med 21: 107-114

63. Newhouse IJ, Clement DB (1988). Iron status in athletes, an update. Sports Med 5: 337-352

64. Newsholme EA (1994). Biochemical mechanisms to explain immunosopression in well-trained and overtrained athletes. Int J Sports Med 15: 142-147

65. Noakes T (1991). Lore of Running. Champaign, IL: Human Kinetics, 408-425

66. O' Connor PJ, Morgan WP, Raglin JS, Barksdale CM, Kalin $\mathrm{NH}$ (1989). Mood state and salivary cortisol levels following overtraining in female swimmers. Physichoneuroendocrinology 14: 303-310

67. Ostrowski K, Hermann C, Bangash A, Schjerling P, Nielsen JN, Pedersen BK (1998). A trauma-like elevation in plasma cytokines in humans in response to treadmill running. $J$ Physiol (Lond) 508:949-953

68. Ostrowski K, Rohde T, Asp S, Schjerling P, Pedersen BK (1998). The cytokine balance and strenuous exercise: TNF-alpha, IL-2beta, IL-6, IL-1ra, sTNF-r1, sTNF-r2, and IL-10. J Physiol (Lond) 515:287-291

69. Pedersen BK, Rohde T, Ostrowski K (1998). Recovery of the immune system after exercise. Acta Physiol Scand 162:325-332

70. Peluso MAM (2003). Alterações de humor associadas à atividade física intensa. Tese (Doutorado)-Faculdade de Medicina, Universidade de São Paulo: São Paulo.

71. Petibois C, Cazola G, Poortmans JB, Deleris G (2002). Biochemical aspects of overtraining in endurance sports: a review. Sports Med 32: 867-878

72. Prada FJA, Voltarelli FA, Oliveira CAM, Gobatto CA, Macedo DV, Mello MAR (2004). Condicionamento aeróbio e estresse oxidativo em ratos treinados por natação em intensidade equivalente ao limiar anaeróbio. Rev Bras Cien Mov 12: 29-34

73. Pyne DB, Baker MS, Fricker PA, MacDonald WA, Telford RD, Weidemann MJ (1995). Effects of an intensive $12 \mathrm{wk}$ training program by elite swimmers on neutrophil oxidase activity. Med Sci Sports Exerc 27: 536-542
74. Rivier A, Pene J, Chanez P, Anselme F, Caillaud C, Pre-Faut C, Godard P, Bousquet J (1994). Release of Cytokines by blood monocytes during strenuous exercise. Int J Sports Med 15:192-198

75. Rossi L, Tirapegui J (1999). Aspectos atuais sobre exercício físico, fadiga e nutrição. Rev Pauli Ed Fís 13:.67-82

76. Seene T, Umnova M, Kaasik P (1999). The exercise myopathy. In: Lehmann M, Foster C, Gastmann U, Keizer H, Steinacker J (ed). Overload, Performance Incompetence and Regeneration in Sport. New York: Kluwer Academic/Plenum Publishers, 119-130

77. Smith JA (1995). Exercise, training, and red cell turnover. Sports Med 19: 9-31

78. Smith JA, Telford RD, Mason IB, Weidemann MJ (1990). Exercise, training and neutrophil microbicidal activity. Int $J$ Sports Med 11: 179-87

79. Smith LL (2000). Cytokine hypothesis of overtraining: a physiological adaptation to excessive stress? Med Sci Sports Exerc 32: 317-331

80. Snyder AC, Jeukendrup AE, Hesselink MKC, Kuipers H, Foster C (1993). A physiological/psychological indicator of overtraining during intensive training. Int J Sports Med 14: 29-32

81. Steinle H (1997). Influence of intensive exercise on sleep and breathing regulation in athletes. Dissertation, University of Freiburg: Freiburg

82. Ullum H, Haahr PM, Diamant M, Palmo J, Halkjaer Kristenses J, Pedersen BK (1994). Bicycle exercise enhances plasma IL- 6 but not change IL-1 $\alpha$ IL- $1 \beta$, IL-6, or TNF$\alpha$ pre-mRNA in BMNC. J Appl Physiol 77:93-97

83. Urhausen A, Gabriel H, Kindermann W (1998). Impaired pituitary hormonal response to exhaustive exercise in overtrained endurance athletes. Med Sci Sports Exerc 30: 407-414

84. Uusitalo AL, Uusitalo AJ, Rusko HK (1998). Endurance training, overtraining and baroreflex sensitivity in female athletes. Clin Physiol 18: 510-20

85. Venkatraman JT, Pendergast D (1998). Effect of the level of dietary fat intake and endurance exercise on plasma cytokines in runners. Med Sci Sports Exerc 30: 1198-1204

86. Verde T, Thomas SC, Shepard RJ (1992). Potential markers of heavy training in highly trained distance runners. $\mathrm{Br} J$ Sports Med 26: 167-175

87. Verma SK, Makindroo SR, Kansal DK (1978). Effect of four weeks of hard physical training on certain physiologi$\mathrm{cal}$ and morphological parameters of basketball players. $J$ Sports Med 18:379-384

88. Vervoon C, Quist AM, Vermulst JM, Erich WBM, DeVries WR, Thussen HH (1991). The behavior of the plasma free testosterona/cortisol ratio during a season of elite rowing training. Int J Sports Med 12: 257-263

89. Yu BP (1994). Cellular defenses against damage from reactive oxygen species. Physiol Rev 74: 139-161.

90. Zavorsky GS (2000). Evidence and possible mechanisms of altered maximum heart rate with endurance training and tapering. Sports Med 29: 13-26

91. Zoopi CC, Antunes-Neto J, Catanho FO, Goulart LF, Motta E, Moura N, Macedo DV (2003). Alterações em biomarcadores de estresse oxidativo, defesa antioxidante e lesão muscular em jogadores de futebol durante uma temporada competitiva. Rev Paul Educ Fís 17: 119-130 